\title{
The new implementation of a computationally efficient modeling tool (STOPS v1.5) into CMAQ v5.0.2 and its application for a more accurate prediction of Asian dust
}

5 Wonbae Jeon ${ }^{1}$, Yunsoo Choi ${ }^{1 *}$, Peter Percell ${ }^{1}$, Amir Hossein Souri ${ }^{1}$, Chang-Keun Song ${ }^{2}$, Soon-Tae $\mathrm{Kim}^{3}$, and Jhoon $\mathrm{Kim}^{4}$

${ }^{1}$ Department of Earth and Atmospheric Sciences, University of Houston, 312 Science \& Research Building 1, Houston, TX 77204, USA

$10 \quad{ }^{2}$ National Institute of Environmental Research, Inchon, Republic of Korea

${ }^{3}$ Division of Environmental Engineering, Ajou University, Suwon, Republic of Korea

${ }^{4}$ Department of Atmosphere Sciences, Yonsei University, Seoul, Republic of Korea

Correspondence to: Yunsoo Choi (ychoi6@uh.edu)

\section{Supplements}

Table S1 is a supplement for the description of difference between the simulated friction velocity and threshold values in the in-line dust module in CMAQ in Sect. 3.1 in the manuscript.

Figure S1 is a supplement for the description of consistency between CMAQ- and STOPS-simulated results in Sect. 4.1 in the manuscript.

Figure S2 is a supplement for the description of difference between standard and alternative emissions used for STOPS simulation in Sect. 4.2.1 in the manuscript. 
Table S1. The averaged friction velocity $\left(u_{*}\right)$ in three land cover categories and threshold friction velocity values $\left(u_{* t i, j}\right)$ for each land cover category used in CMAQ_Dust simulation.

\begin{tabular}{c|cc}
\hline Land Cover Categories & $u_{*}$ & $u_{*}$ (CMAQ_Dust) \\
Shrubland & 0.23 & 1.54 \\
Mixed Shrubland-Grassland & 0.16 & 0.55 \\
Barren or Sparsely vegetated & 0.18 & 0.65 \\
\hline
\end{tabular}



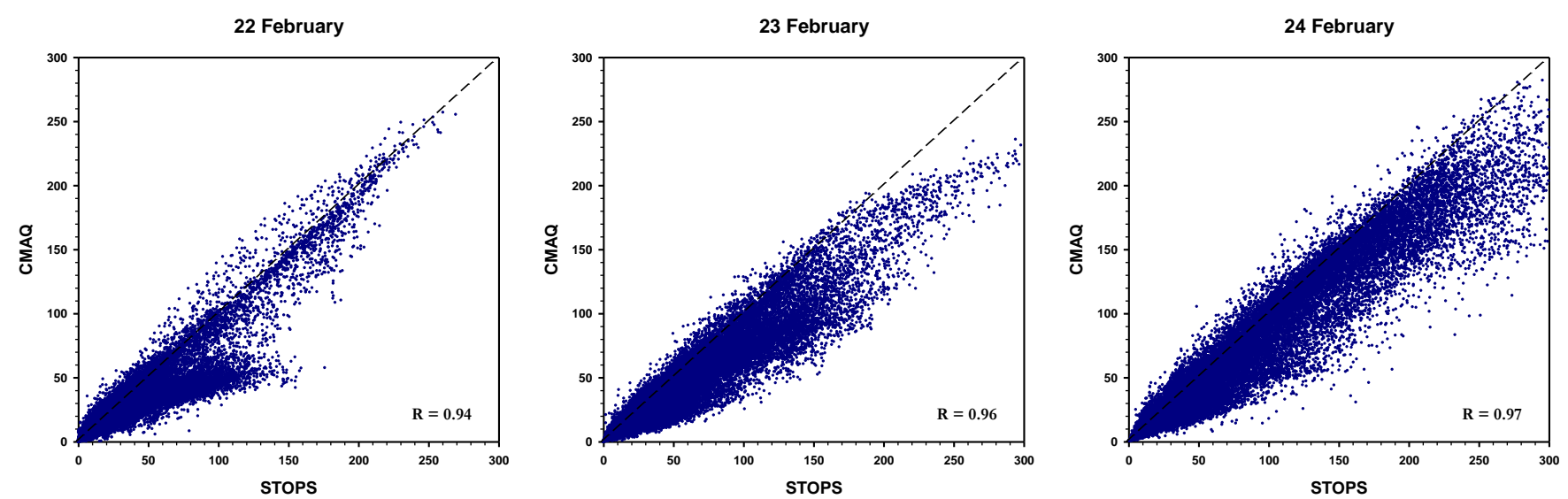

Figure S1. Scatter plots between STOPS- and CMAQ-simulated $\mathrm{PM}_{10}$ concentrations during the Asian dust events. The correlation coefficients (R) appear in the bottom-right of each plot. 

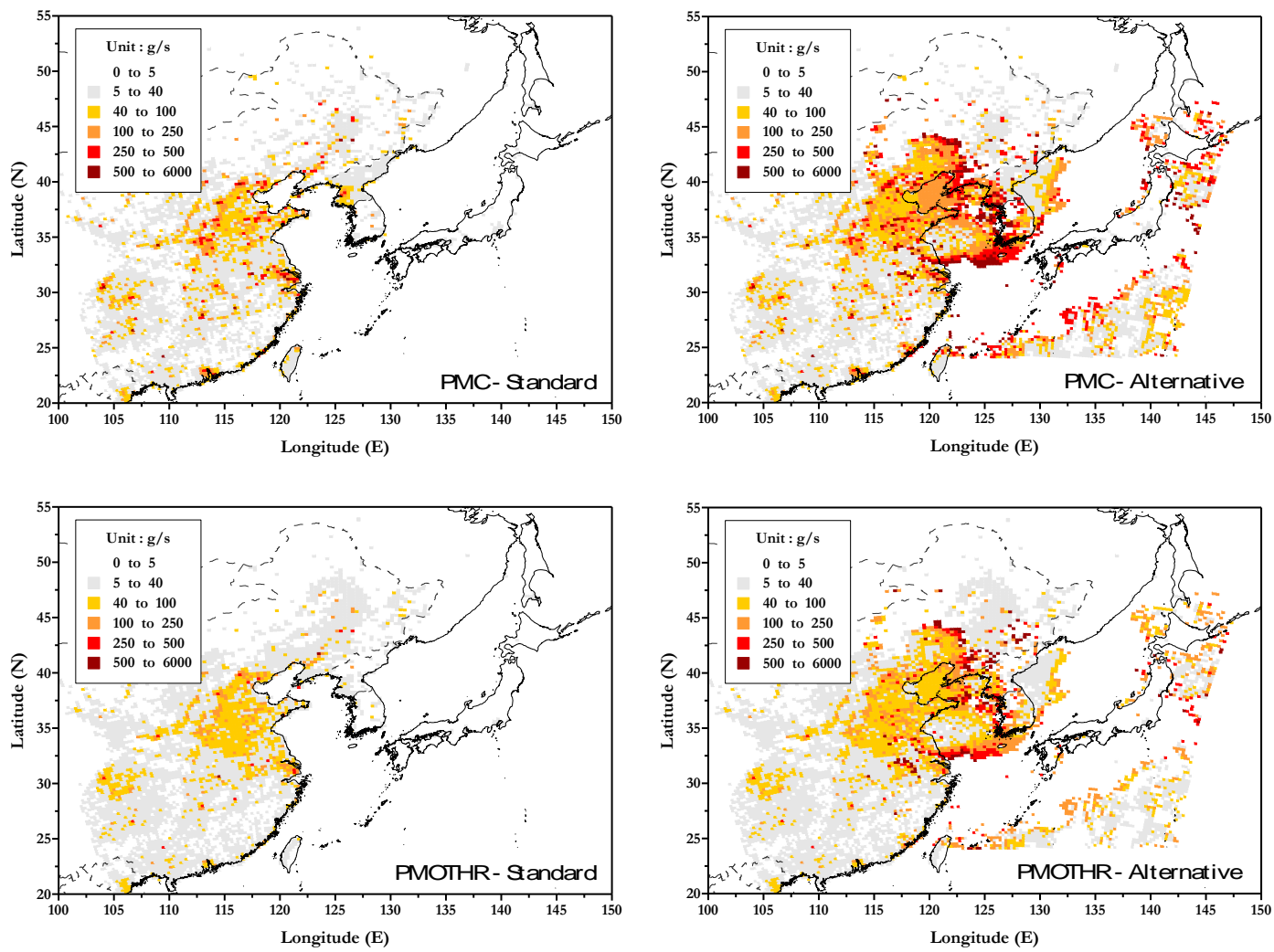

Figure S2. Difference between the emissions rates $\left(\right.$ grams second $\left.^{-1}\right)$ of standard and alternative emissions (to represent enhanced GOCI AOD) data. The PMC and PMOTHR denote coarse and unspeciated fine particles, respectively. 